\title{
Georges Bertrand, un géographe interroge l'agronomie
}

\section{Propos recueillis par Daniel Terrasson}

\author{
Georges Bertrand ${ }^{\mathrm{a}}$, Daniel Terrasson ${ }^{\mathrm{b}}$ \\ a Professeur émérite de géographie, Université de Toulouse-Le Mirail, Maison de la Recherche, GEODE - UMR 5402 CNRS, \\ 5 allée Antonio Machado, 31058 Toulouse cedex 1, France, \\ b Agronome, CEMAGREF, Département Gestion des territoires, 50 avenue de Verdun, 33612 Cestas cedex, France
}

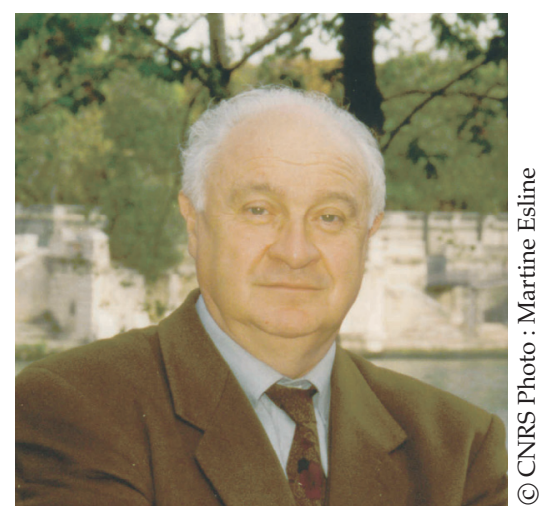

Daniel Terrasson : Georges Bertrand, vous êtes géographe et vous avez abondamment contribué au débat sur l'interdisciplinarité. Dans l'ouvrage que vous avez publié en 2002, Une géographie traversière : l'environnement à travers territoires et temporalités ${ }^{1}$, vous évoquez à plusieurs reprises vos échanges avec les écologues, avec les chercheurs des sciences de l'homme, mais vous parlez très peu des agronomes. Par contre, vous êtes intervenu en 2002 aux entretiens du Pradel consacrés à la confrontation des agronomes avec la question du territoire. Pouvez-vous nous expliquer comment vous avez rencontré l'agronomie et nous dire ce qu'elle vous a apporté?

Georges Bertrand : Géographe de formation, et de profession, j'ai heureusement reçu, pendant près d'un demi-siècle, de multiples et salutaires « chocs conceptuels » en provenance des disciplines voisines, parmi lesquelles l'agronomie.

Auteur correspondant: G. Bertrand, gbertran@univ-tlse2.fr

1 Bertrand, G. et C., 2002. Une Géographie traversière : l'environnement à travers territoires et temporalités, Paris, Arguments.
Ce fut d'abord, pendant les années 1956-1958, alors quej'étais étudiant à l'Institut de géographie de Toulouse, mon premier contact avec Olivier de Serres à travers ses ouvrages et surtout l'enseignement rayonnant du doyen Daniel Faucher. Au-delà des structures agraires, déjà rabâchées, s'ouvrait la dynamique humaine du «mesnage des champs », doublement annonciatrice de l'agronomie moderne et de l'aménagement des territoires.

Le second choc, décisif du point de vue méthodologique, fut la découverte, presque fortuite, entre 1961 et 1962, du concept de «profil cultural » de Stéphane Hénin (Encadré). Autodidacte en pédologie, initié à l'analyse des horizons pédologiques sous forêts et landes selon la méthode de Philippe Duchaufour, ce fut mon premier contact avec la réalité des sols remaniés par les techniques et les pratiques agricoles, dont la fameuse «semelle de labour » qui m'avait posé tant de problèmes sur le terrain. Le concept de «profil cultural » m'est apparu d'emblée comme une révolution conceptuelle. Il surmontait, sur le terrain, sans contorsions épistémologiques ni méthodologiques, l'opposition théorique nature/culture (que l'on traîne encore aujourd'hui). Cette notion de corps hybridés soulignait, dès les années 1960, le porte-à-faux des sciences de la nature par rapport aux sciences de la société et, pour ma pratique, celui de la géographie physique par rapport à la géographie humaine. Le "profil cultural » est l'une des sources d'inspiration du concept de géosystème et, plus tardivement, du système GTP (Géosystème-Territoire-Paysage).

À partir des années 1970, les contacts se sont multipliés avec les agronomes, zootechniciens et forestiers, plus spécialement avec ceux de 1'Inra-Sad, avec lesquels j'ai beaucoup d'affinités épistémologiques, et avec 


\begin{abstract}
Encadré. Le profil cultural
Le profil cultural est un concept développé par Stéphane Hénin dans l'ouvrage éponyme : Hénin, S., Gras, R., Monnier, G., 1960. Le profil cultural. Principes de physique $d u$ sol, Paris, SEIA. Nul mieux que J.-P. Deffontaines n'en a souligné l'importance :

"Hénin propose un concept opératoire, appuyé sur une théorie de l'évolution des sols sous culture et permettant d'étudier globalement l'ensemble fonctionnel constitué par le peuplement végétal, le sol, le climat et les interventions de l'homme. L'analyse du profil cultural fournit à l'agronome le moyen de lire sur une tranche de sol l'histoire des pratiques appliquées par un agriculteur à une parcelle, de juger l'effet de ces pratiques et de définir des règles d'action. La méthode est neuve [...] elle apparaît systémique avant l'heure. .. »

(source : Les Sentiers d'un géoagronome, Paris, Arguments, 1998, p. 117)
\end{abstract}

tous ceux que j'ai rencontrés dans les comités nationaux (ECV : Espace et Cadre de Vie; ECAR : Écologie et Aménagement Rural; DMDR : Diversification des Modèles de Développement Rural) ou des programmes interdisciplinaires (PIR-CNRS, Élevage dans les Pyrénées DGRST, Paysages et politiques publiques du ministère de l'Écologie et du Développement durable), sans compter les contacts avec des agronomes et forestiers étrangers (Espagne, Venezuela, Colombie).

L'agronome Jean-Pierre Deffontaines, naturellement enraciné dans la géographie, a toujours joué un rôle éminent dans ce dispositif. La parution de l'ouvrage Les Sentiers d'un géoagronome $e^{2}$ ravive et surtout repositionne avec beaucoup de pragmatisme le débat sur l'interdisciplinarité et l'hybridation des concepts. Les agronomes sont de plus en plus confrontés à la mobilité des territoires et à l'émergence de la dimension environnementale. JeanPierre Deffontaines nous propose la définition suivante de la géoagronomie : "Ce champ se rattache à la géographie par les objets d'étude, les structures spatiales, la dynamique des phénomènes et les activités dans le territoire. Mais son analyse est dans l'agronomie car les facteurs de structuration du territoire sont recherchés dans le fonctionnement et la dynamique des systèmes techniques aux différents niveaux où s'organise l'activité agricole. » La géoagronomie est une façon parmi d'autres, mais particulièrement pertinente, d'entrer dans cette nouvelle problématique. La question, à la fois épistémologique et méthodologique, est moins de passer une frontière entre deux disciplines que d'exploiter la réalité même de la frontière pour en faire un lieu de rencontre et d'élaboration d'un nouveau corpus méthodologique entre discipline et interdiscipline.

\footnotetext{
${ }^{2}$ Deffontaines, J.-P., 1998. Les Sentiers d'un géoagronome, Paris,
} Arguments.
Après le grand bond de l'interdisciplinarité tous azimuts auquel nous avons tous participé, il y a comme une insatisfaction et un vide. Les questions vives qui se dessinent et les méthodes pour les aborder nous renvoient aux disciplines, mais les débordent toujours autant. Se pose alors de façon concrète la mise en forme de systèmes transversaux, circum-disciplinaires, bien circonscrits, construits et finalisés, sinon permanents, du moins dotés d'une certaine durabilité. Il les faut plus aisés à mettre en œuvre et surtout plus efficaces que les enchevêtrements interdisciplinaires traditionnels. La géoagronomie, dont J.-P. Deffontaines nous propose de suivre les premiers sentiers, ne défriche-t-elle pas un nouveau territoire de recherche qui, tout à la fois, transcende les deux disciplines concernées et contribue à discipliner la prolixité interdisciplinaire? Ce pari, qui ne peut que nous enrichir, mérite d'être relevé.

D.T. : Vous évoquez l'interdisciplinarité, la manière dont celle-ci renvoie aux disciplines; pouvons-nous maintenant revenir sur cet aspect? Comment voyez-vous les rapports entre agronomie et géographie?

Georges Bertrand : L'agronomie est, consubstantiellement, une science doublée d'un art qui s'enracine dans le territoire. La parcelle agricole, lieu des techniques et des pratiques, en est l'unité fonctionnelle de base et, souvent, la référence privilégiée, voire unique. Le tout est de sortir de la parcelle. Or, l'agronomie contemporaine a un problème existentiel avec un territoire devenu plus urbain que rural et plus rural qu'agricole. La parcelle et l'exploitation agricoles, les terroirs, ainsi que toutes les formes d'emprises agricoles, ne sont plus que des éléments parmi d'autres d'une mosaïque géographique morcelée et instable, soumise à de multiples stratégies économiques et valeurs culturelles contradictoires. L'agronome est de moins en moins seul sur sa parcelle. Il lui faut s'ouvrir à la complexité du territoire.

Le retour, et le recours, au territoire n'est pas univoque. Il exprime des cas de figure multiples et contradictoires. S'agit-il d'un repli frileux sur une valeur refuge : le champ bucolique? S'agit-il de rompre le fil de la filière et d'une fuite en avant, à contre-filière? S'agit-il d'une avancée raisonnée dans la tradition de l'agronomie classique? Ou bien s'agit-il des prémisses d'une révolution copernicienne, annonciatrice d'une nouvelle forme d'agronomie, en quête de redéploiement scientifique et, plus encore, d'ouverture culturelle, à la recherche d'une meilleure adéquation avec la globalisation et la mondialisation des questions sociales et environnementales ? Il y a certainement un peu de tout cela : du technologique et du culturel, de la méthode et de la pratique, du patrimoine et du prospectif, de l'agronomique et de l'interdisciplinaire. Dont du géographique.

La géographie, qui se définit généralement comme une "science du territoire», est-elle, pour une fois, en première ligne? Y aurait-il avec l'agronomie une certaine 
convergence épistémologique qui pourrait augurer d'une future confluence conceptuelle et méthodologique? Dans une sorte d'entre-deux disciplinaire. Sans pour autant se couper des autres disciplines. Vers une "science diagonale » au sens de R. Caillois. Mais ici le but final importe moins que l'intention et la démarche.

$\mathrm{Au}$ centre du débat, il y a le territoire. Mais de quel territoire s'agit-il ? L'ambiguïté de ce terme n'a cessé de croître. Il s'emmêle, sans se superposer, avec les notions d'espace, d'environnement, de paysage, voire avec des concepts scientifiques tels que l'écosystème, le géosystème, l'agrosystème. Je n'entrerai pas dans des querelles byzantines. Le territoire ne se taille pas à la mesure d'une discipline, voire d'un objectif particulier. Il n'y a pas plus un territoire de l'agronome qu'il n'y a un territoire du géographe. Seul existe le territoire des hommes aujourd'hui dilaté à la planète entière. Avec, à la base, cette nouvelle donne qui constitue le leitmotiv de mon propos: nulle part l'agricole, voire le rural, n'est l'unique moteur, et cela, jusqu'à l'échelle de la moindre parcelle ou du moindre troupeau. D'où cette affirmation de B. Vissac ${ }^{3}$ que je fais mienne : "Notre questionnement est celui de la contribution de l'agriculture au développement d'un territoire plutôt que du développement de l'agriculture dans un territoire. » $C^{\prime}$ est déjà une première approche de la géoagronomie.

D.T. : Vous nous présentez le territoire comme point de convergence entre ces deux disciplines, issu d'une évolution récente des problématiques. Mais n'y avaitil pas des prémisses qui permettaient d'anticiper cette émergence?

Georges Bertrand : L'analyse des rapports historiques entre les deux disciplines est ici hors de propos ${ }^{4}$. Par contre, il est indispensable de mettre en perspective la prise en considération commune du "géographique». Par cet adjectif substantivé, je désigne cette dimension d'essence territoriale dont la géographie s'est fait une spécialité, mais qui n'a jamais été absente de l'agronomie.

Jusqu'au milieu du XXe siècle, la campagne française, sous les apparences d'un "ordre éternel des champs », a constitué un socle territorial plus ou moins stable et un patrimoine scientifique commun à plusieurs générations de chercheurs. Quelques noms parmi d'autres peuvent être cités : M. Bloch, P. Deffontaines, A. Demolon, R. Dion, D. Faucher, G. Haudricourt, L. Febvre, A. Meynier, etc. Les thèmes abordés font la part belle au territoire, à son organisation, à sa mise en valeur : structures agraires des finages et parcellaires des terroirs, techniques et pratiques agricoles, analyses des différentes « révolutions

\footnotetext{
3 Vissac, B., 1989. Quand les agronomes s'en vont aux champs, in Marchal, J.-Y., L'Espace géographique, 3, (1990-1991), 217.

4 Bertrand, C. et G., 1975. Pour une histoire écologique de la France rurale, in Duby, G., Wallon, A., Histoire de la France rurale, Paris, Le Seuil, vol. 1, 35-116.
}

agricoles ». Avec le souci minutieux du terrain et de l'enquête, au plus près des réalités humaines. L'agronomie française était imprégnée d'une culture générale historico-géographique très territorialisée et très régionalisée qu'elle a en grande partie perdue. À ce jour, demeurent de nombreuses filiations qui ne sont pas seulement professionnelles.

Les Trente Glorieuses marquent, sinon une rupture, du moins une distension des liens. Paradoxalement, alors que l'aménagement du territoire est au cœur de la reconstruction économique et sociale, on assiste à un double mouvement de remembrement brutal du territoire et à un démembrement des disciplines et des recherches. De plus en plus artificialisé et homogénéisé, le territoire est géré sur des bases productivistes de court terme. L'indispensable approfondissement des disciplines et l'apparition de nouvelles spécialisations concourent au compartimentage des interprétations scientifiques. Les technologies, de plus en plus "avancées », tendent à se substituer à la méthode et l'épistémologie est passée de mode. L'émergence en France, vers les années 1970, de l'écologie de synthèse ne corrige que très partiellement ce parcellement des savoirs agronomiques, dans la mesure où l'écosystème, d'essence naturaliste, s'arrête souvent sur les marges de l'espace cultivé 5 . Pendant ce même temps, la géographie s'épuise en débats internes et se morcelle. La géographie physique s'étiole. La géographie rurale devient une branche de la géographie sociale, dans l'ombre de la ville.

Aujourd'hui l'interdisciplinarité, en dépit de ses flottements ${ }^{6}$, a rapproché les hommes et les idées. Le territoire, si malmené, est de retour. Après le temps des filières, s'agit-il du temps des territoires? Substitution et/ou complémentarité?

D.T. : Vous nous avez montré les raisons d'une convergence entre agronomie et géographie, vous nous avez rappelé le rôle central du territoire pour la géographie. En tant que géographe, quelles questions majeures souhaitez-vous poser plus particulièrement aux agronomes?

Georges Bertrand : Edgard Morin rappelle que «la complexité est à la base», Michel Sebillotte évoquait le vertige de la complexité. Nous n'avons plus le choix. Les problèmes que les agronomes se posent et que les nonagronomes leur posent transgressent de plus en plus largement le champ traditionnel de l'agronomie. En fait, ils n'ont plus de frontières. Tout en développant de profondes racines agronomiques. Parmi ces questions multiformes et toujours enchevêtrées, je n'en retiendrai que

\footnotetext{
${ }^{5}$ Hubert, B., 2002. Les recherches sur le Méjan : les disciplines au cœur des tensions entre science et politique, Natures Sciences Sociétés, 10, 2, 67-69.

${ }^{6}$ Legrand, P., 1998. Repères dans le paysage agricole français, Le Courrier de l'environnement de l'INRA, 33, 81.
} 
cinq qui portent directement sur le territoire et n'épuisent pas le sujet.

Tout d'abord, on est confronté à des natures pas très naturelles, mais avec une naturalité omniprésente.

Il n'y a pas de territoire et, pratiquement, d'agriculture sans terre, sans cette part de naturel qu'impliquent le vivant et les grands cycles biogéochimiques. Les catastrophes naturelles et les changements et/ou oscillations climatiques, l'épuisement de certaines sources naturelles, sont là pour nous le rappeler. Pourtant, la question de la nature au quotidien ne paraît plus essentielle. Elle est occultée par l'économicisme ambiant et, paradoxalement, masquée par l'émergence des toutes-puissantes biotechnologies.

De fait, il est de plus en plus difficile de faire « un bon usage de la nature ${ }^{7}$ ». Après avoir surmonté un déterminisme naturel honteux ${ }^{8}$, il faut aujourd'hui dépasser les réticences, effacer les lacunes en évitant le mélange des genres. Entre les conceptions métaphysiques de la Nature, opposables à l'Homme, et les processus biophysiques qui fondent les productions végétales et animales conduites par les sociétés humaines, il y a de multiples transitions et manières d'aborder le sujet. Avec différentes combinatoires entre faits naturels et faits sociaux. L'anthropisation tend à devenir le processus dominant. . . mais une part de nature persiste toujours. Le concept de naturalité, tel qu'il est avancé par J. Lecomte ${ }^{9}$, mérite d'être approfondi : «[... ] la naturalité d'un système écologique s'apprécie le long d'un gradient. La véritable naturalité peut être estimée en fonction de l'influence des activités humaines sur l'évolution du système considéré. » La recherche de modèles d'anthropisationartificialisation doit être systématisée à toutes les échelles temporospatiales. C'est un grand pas de plus sur la voie déjà tracée $\mathrm{du}$ « profil cultural ».

En second lieu, l'espace agricole balance entre «la fin de l'agrosystème » et un certain « renouveau des terroirs ».

La mosaïque agricole, avec ses parcelles, ses terroirs et ses pays, fonctionnait sur la base d'un système territorial généralisé et organisé, sinon indépendant, du moins autonome. Aujourd'hui, cet agrosystème tend à devenir une sorte de kaléidoscope agité par d'incessantes secousses, agricoles et non agricoles, et dévoré par de plus en plus larges empiétements urbains. Tout en entretenant encore la plus grande partie du territoire - avec difficulté, il est vrai -, l'agriculture est repoussée sur les marges du système économique et social ainsi que du système écologique. Son territoire est de moins en moins un agrosystème auto-organisé et cohérent. Comme le déplorent déjà B. Hervieu et E. Pisani dans Le Monde du 12 mars

\footnotetext{
7 Larrère, C. et R., 1997. Du bon usage de la nature, Paris, Aubier.

8 Bertrand, C. et G., op. cit.

9 Lecomte, J., 1999. Réflexions sur la naturalité, Le Courrier de l'environnement de l'INRA, 37, 5-10.
}

1996, « aux territoires succèdent des bassins céréaliers, porcins, allaitants, laitiers qui font de nos territoires un puzzle aux pièces disparates ». Cette rupture du continuum territorial et de sa logique fonctionnelle est lourde de conséquences écologiques, économiques, voire psychologiques : rupture ou dénaturation des stocks et des flux de matière et d'énergie, difficultés d'exploitation, isolement et « ensauvagement ».

Les contraintes imposées aux agrosystèmes traditionnels n'annoncent pourtant pas la «fin des terroirs». Si certains de ceux-ci s'effacent, remplacés par la friche, le béton ou le bitume, d'autres résistent, se consolident ou se créent autour de productions de qualité. Les terroirs viticoles ont depuis longtemps donné l'exemple. Les « terroirs de reconquête » se multiplient et les chercheurs retrouvent l'importance de «la composante physique de $1^{\prime}$ effet terroir ${ }^{10}$.

En troisième lieu, il ne faut pas se tromper de paysage : le paysage-territoire est à la fois paysage-décor et paysage-outil.

L'irruption du paysage et des valeurs esthétiques et patrimoniales qui lui sont attachées bouleverse notre vision du territoire. Ce n'est qu'un début. Du territoire, le paysage n'est pas seulement l'apparence. Il en constitue l'architecture matérielle, visible par tous, et il en exprime la permanence patrimoniale. Il est la mémoire longue des campagnes. Dimensions un peu trop oubliées par l'agronomie classique qui s'est focalisée sur la production dans l'espace et sur l'espace de production.

Le paysage tel qu'il est aujourd'hui perçu et vécu exprime une sorte de revanche culturelle d'origine urbaine contre les excès d'un désenchantement et d'une laïcisation du territoire. L'angélus de Millet tinte à nouveau sur la campagne française, symbole d'un pseudoretour à une pseudo-nature. Même agrémenté, ce paysage agricole n'est pas un jardin et il ne relève pas de techniques jardinatoires. Le paysagisme n'est qu'une des nouvelles dimensions d'un territoire qui demeure encore pour longtemps dessiné par la production et qui doit à cette dernière son équilibre d'artifice et son charme d'artefact. Si les paysages extraordinaires méritent une attention particulière, ils ne doivent pas masquer la multitude des paysages dits « ordinaires » qui constituent, de fait, l'extraordinaire harmonie et diversité des campagnes ${ }^{11}$.

La quatrième question porte sur la biodiversité et la géodiversité territoriales : à travers les géosystèmes et les jeux d'échelle temporospatiaux.

\footnotetext{
10 Dorioz, J.-M., et al., 2000. La composante milieu physique dans l'effet terroir pour la production fromagère, Le Courrier de l'environnement de l'INRA, 40, 47-55.

11 Lelli, L., 2000. Le Paysage ordinaire : l'exemple du NordComminges (Haute-Garonne, France). Essai méthodologique et pratique, Thèse de doctorat, Géode - Université de Toulouse-Le Mirail.
} 
Dès les années 1950, alors que le botaniste H. Gaussen stigmatisait l'absence de "dimension biologique » en géographie, le géographe $\mathrm{D}$. Faucher définissait, dans l'introduction de sa Géographie agraire, «l'agriculture comme une biologie ». Avec l'appui grandissant de la biologie et de l'écologie, l'agronomie est devenue l'une des grandes sciences du vivant. L'écosystème a bien rempli sa mission. Mais l'agronome ne peut en rester là. Il est en permanence confronté à la complexité spatiale et temporelle du territoire : relief, climat, eaux courantes ou stagnantes, sols, qui interfèrent au sein du milieu cultivé. Les éléments abiotiques sont en interaction permanente avec les composantes biotiques et anthropiques. L'agrosystème est un géosystème tronqué et modifié pour produire une récolte ${ }^{12}$. La combinaison entre tous ces éléments change dans le temps et dans l'espace. Pour maîtriser ce jeu d'échelle essentiel à la compréhension de l'espace agricole et rural, l'agronome doit s'extraire de la parcelle et de l'exploitation agricole. Il lui faut, plus que jamais, appréhender la structure et le fonctionnement des systèmes territoriaux à toutes les échelles d'espace et de temps.

Enfin, pour le cinquième et dernier point, le temps et les états du territoire doivent être placés au centre du débat.

La durabilité ne sera qu'un mot-valise tant que les chercheurs n'auront pas analysé les multiples temporalités du territoire : durées, périodisations, rythmes, y compris crises et catastrophes. Le territoire n'existe concrètement, aussi bien pour l'agriculteur que pour le scientifique, qu'à travers ses états (instantanés, quotidiens, saisonniers, annuels, interannuels, etc.) et leur succession. Les journées du PIREVS (Programme interdisciplinaire de recherche Environnement, vie et sociétés) du CNRS (Toulouse, 1998) font le point des recherches sur les temps de l'environnement et leurs répercussions sur le fonctionnement des territoires ${ }^{13}$. En concevant, a priori, le territoire comme un espace-temps, on évite l'écueil de références temporelles disparates puisées dans d'autres disciplines. Des notions de base de l'agronomie, telles que la potentialité, la fertilité, la ressource, peuvent être ainsi recadrées dans un territoire donné, pour un système de production donné et dans un temps donné, en fonction d'une société donnée. Il reste à l'agronome à se doter d'un outil territorial efficace.

D.T. : Dans ce contexte, vous nous avez proposé d'emprunter les sentiers de la géoagronomie; quelles conséquences cette voie impose-t-elle pour les pratiques de recherche en agronomie?

\footnotetext{
12 Bertrand, C. et G., op. cit.

13 Barrué, M, Bertrand, G. (Eds), 1997. Les Temps de l'environnement, Journées du PIREVS, Toulouse, Presses universitaires du Mirail ; Bertrand, C. et G., Le géosystème : un espace-temps anthropisé. Esquisse d'une temporalité environnementale, ibidem, 65-76.
}

Georges Bertrand : J'évoquais la perspective d'une «science diagonale». L'idée est utopique. Il ne s'agit que de se projeter au plus loin pour agir au plus près. L'inventaire des questions vives, pourtant très incomplet, nous laisse en présence de fragments de territoires (parcelles, terroirs, bassins, paysages, espaces dits naturels...) et d'éléments spatiaux disparates (sols, végétation naturelle ou cultivée, eaux... ). Comment rendre compte, dans un même mouvement, de l'unité du fait territorial et de son caractère multifonctionnel dans le temps et dans l'espace? N'est-ce pas changer le vertige de M. Sebillotte en mirage?

La question n'est pas seulement de l'ordre de la méthode mais d'un paradigme, bien plus large, comme le recommande E. Landais ${ }^{14}$, qu'un simple «paradigme expérimental ». Si ce mot effraie par son ambition, parlons humblement de «biais », comme le berger de A. Leroy qui ajuste la gestion de son troupeau et de son pâturage à partir de techniques et de pratiques toujours réinventées ${ }^{15}$. Pour valoriser au mieux le territoire, je propose au débat un système d'analyse à trois niveaux : épistémologique, méthodologique, didactique.

Premièrement, il faut élargir le champ épistémologique : du «profil cultural » au profil culturel. Des publications vivifiantes, par exemple dans Le Courrier de l'environnement de l'INRA, viennent secouer l'édifice agronomique et proposer de nouvelles voies de réflexion, autocritiques et ouvertes sur une interdisciplinarité réévaluée. Ce réveil épistémologique concerne très directement le rôle et la place de l'agronome sur le territoire. La première urgence est, $\mathrm{y}$ compris pour les non-agronomes, de définir un nouveau champ sémantique et conceptuel qui ne sera pas sans rappeler le débat critique livré, il y a quelques décennies, autour de la notion de fertilité. Toute l'approche environnementale du territoire est concernée et aucune discipline en particulier n'en possède les clés. Par exemple, que signifient ressource naturelle, potentialité, durabilité, irréversibilité, renouvelabilité, etc., hors de tout système de références socio-culturel enraciné dans un espace-temps donné ? S. Hénin, en proposant le profil cultural comme l'interface agronomique entre le naturel et le social, a définitivement changé le regard de l'agronome sur le champ cultivé. Aujourd'hui, les évolutions-révolutions subies à la fois par l'agriculture et par le territoire nous amènent à concevoir des outils qui embrassent le monde non agricole et non agronomique. Sans oublier les spécificités des pays du Sud. La littérature agronomique, celle des sciences sociales, voire la littérature scientifique «dure», ne suffisent plus. «Composer demande une tension entre local et global, voisin et lointain, récit et règle, l'unicité du verbe et le pluralisme inanalysable des sens, monothéisme et paganisme,

\footnotetext{
14 Landais, E., 1998. Agriculture durable et nouveau contrat social, Le Courrier de l'environnement de l'INRA, 33, 5-22.

15 Deffontaines, J.-P., op. cit., 157.
} 
l'autoroute internationale et les villages retirés, la science et les littératures ${ }^{16}{ }$.

Ensuite, il faut proposer une méthode multidimensionnelle pour appréhender le territoire tel qu'en luimême : avec, par exemple, la contribution du système tripolaire GTP ${ }^{17}$.

Les analyses sectorielles, de plus en plus spécialisées et finalisées, fournissent une masse envahissante de faits d'observation et de résultats d'expérimentations. Pour aussi indispensables qu'elles soient, elles ne peuvent suffire à saisir la structure et le fonctionnement interactifs du territoire. Si elles font office de connaissance des processus, elles ne s'élèvent jamais jusqu'à l'intelligence du système-territoire. L'écosystème constitue le meilleur exemple de concept rassembleur et téléologique concernant le monde vivant. Toutefois, il ne peut exprimer toute la complexité-diversité du territoire, que ce soit dans ses aspects abiotiques (relief, modelés, climat. . .), socioéconomiques ou, à plus forte raison, culturels (artialisation, patrimonialisation... ). De plus, il faut prendre en compte la diversité des approches et des finalités qui se développent et souvent s'opposent sur un même territoire. La méthode ne peut se fonder sur un concept unique et univoque. D'où la proposition de traiter du territoire à travers un système tripolaire qui ménage trois entrées principales dans un même territoire (il peut y en avoir d'autres) :

- le géosystème-source traitant des objets et des processus biophysiques qui, pour l'essentiel, sont déjà plus ou moins anthropisés ;

- le territoire-ressource qui prend en compte les structures et les fonctionnements liés aux activités socioéconomiques, compte tenu des données géosystémiques et paysagères ;

- le paysage-ressourcement appréhendant la dimension sensible et symbolique au travers des représentations socioculturelles.

À ces trois concepts ou notions de base correspondent trois grilles spatiotemporelles spécifiques, sans emboîtement de principe, ce qui permet de mettre en évidence les contradictions, discordances et déphasages qui sont à l'origine de conflits territoriaux où l'agriculture a sa part et que l'agronome doit contribuer à démêler.

Une telle démarche scientifique, aux frontières de l'agronomie et de la géographie, permet de situer l'activité agricole et l'intervention de l'agronome dans les mouvements de plus en plus saccadés des sociétés et des territoires.

Enfin, il faut développer une didactique du territoire.

Cet élargissement de la pensée et de la méthode est déjà largement amorcé grâce aux efforts pédagogiques engagés, en particulier par l'enseignement agricole.

\footnotetext{
16 Serres, M., 1983, Les Cinq Sens, Paris, Grasset, 262.

17 Barrué, M., Bertrand, G. (Eds), op. cit.
}

Celui-ci a intégré la double dimension économique et écologique, désormais indissociable dans la problématique environnementale et, encore plus nettement, dans celle du développement durable qui commence à prendre de mieux en mieux en compte les agronomies des pays du Sud. La dimension territoriale n'est pas absente, mais elle n'est traitée qu'au travers de la production et de la productivité (quand ce n'est pas du productivisme). L'analyse naturaliste des géosystèmes anthropisés et l'analyse socioculturelle des représentations paysagères sont en train de faire évoluer les contenus traditionnels de l'enseignement agronomique. C'est le cas des travaux exemplaires de Y. Michelin ${ }^{18}$ à l'ENITA de Clermont-Ferrand

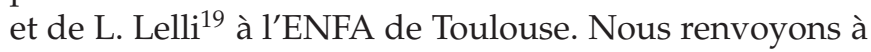
leurs travaux pionniers.

L'extrait des Sentiers d'un géoagronome de J.-P. Deffontaines, cité précédemment, trace des perspectives qui ne concernent pas seulement l'agronomie. Il reste à prolonger cette proposition en l'ouvrant encore davantage. La question n'est pas de ramener sur l'agronomie toutes sortes d'apports extérieurs. C'est de permettre à l'agronomie de sortir de l'agronomie, de se reconstruire et de se redéployer vers de nouveaux horizons... qui ne sont pas tous géographiques. Sans rien perdre de la dimension agronomique. «S'agissant [... ] de la gestion de l'espace rural, moins que jamais, on ne peut prétendre la penser à travers les seuls systèmes techniques, mais on ne peut pas non plus la penser sans $e^{20}{ }^{20} »$.

"L'agronomie est déjà une science interdisciplinaire mais est-ce la bonne?»Cette interrogation de D. Moriss ${ }^{21}$, teintée d'humour britannique, doit, elle aussi, être prolongée, «... mais est-ce la bonne... » pour affronter les questions territoriales et environnementales de demain, celles des pays du Sud comme celles des pays du Nord?

La géoagronomie, soucieuse à la fois de dimension territoriale et de dimension sociale et culturelle, n'est peut-être pas la bonne réponse, et surtout pas la seule. Mais elle est l'une des bonnes questions à poser, tout particulièrement aux agronomes et aux géographes, à un moment où ces disciplines s'interrogent, ou devraient s'interroger sur l'efficacité aussi bien de leurs méthodes propres que sur l'interdisciplinarité telle qu'elle est pratiquée aujourd'hui.

\footnotetext{
18 Michelin, Y., et al., 2002. Le Paysage dans un projet de territoire. Démarche et méthode expérimentées en Limousin, Chambre d'agriculture de la Haute-Vienne.

19 Lelli, L., op. cit.

20 Osty, P.-L., et al., 1998. Comment analyser les transformations de l'activité productrice des agriculteurs? Propositions à partir des systèmes techniques de production, Étud. Rech. Syst. Agraires, Dev. INRA, 31, 397-413.

${ }_{21}$ Moriss, D., 1998. La place de l'agronomie dans la recherche environnementale, Les Dossier de l'environnement de l'INRA, 17, 67-70.
} 